\title{
Effect of different reversed micelles on autooxidation and photooxidation of stripped corn oil
}

\author{
By Y.A. El-Shattory ${ }^{1}$, M.A. Saadia ${ }^{1}$, S. F. Hamed ${ }^{1}$ and K. Schwarz ${ }^{2}$ \\ ${ }^{1}$ Fats and Oils lab, National Research Centre, Tahrir st., Dokki, Cairo, Egypt. \\ ${ }^{2}$ Institut für Humanernährung und Lebensmittelkunde, Max-Eyth Str. 6, D-24098 Kiel, Germany.
}

\section{RESUMEN}

Efecto de diferentes miscelas en la autooxidación y fotooxidación de aceite de maíz libre de antioxidantes.

El efecto de miscelas inversas (RMs) en combinación con $\alpha$ tocoferol y Trolox fue evaluado en la autooxidación y fotooxidación de aceite de maíz libre de antioxidantes. La oxidación fue seguida por la medida de la formación de hexanal e hidroperóxido. Las RMs influyeron en la estabilidad de la oxidación del aceite dependiendo del tensioactivo usado. Las RMs de lecitina causaron una mayor disminución en la oxidación que las RMs de poligliceril-3oleato y las RMs de metilglucosa dioleato. Todas las RMs redujeron la inhibición de la oxidación cuando se añadieron en combinación con antioxidantes externos.

PALABRAS-CLAVE: Aceite de maíz - Autooxidación - Fotooxidación - Lecitina - Metil glucosa dioleato - Miscelas inversas - Oxidación - Poligliceril-3-oleato.

\section{SUMMARY}

Effect of different reversed micelles on autooxidation and photooxidation of stripped corn oil .

The effect of reversed micelles (RMs) in combination with $\alpha$-tocopherol and Trolox was evaluated on the autooxidation and photooxidation of stripped corn oil. Oxidation was followed by measuring hydroperoxide and hexanal formation. RMs affected the oil oxidation stability to a degree depending on the surfactant used. Lecithin RMs caused a stronger decrease in oxidation than polyglyceryl-3-oleate RMs and methylglucose dioleate RMs. All RMs reduced the inhibition of oxidation when added in combination with external antioxidant.

KEY-WORDS: Autooxidation - Corn oil -Lecithin - Methyl glucose dioleate - Oxidation - Photooxidation - Polyglyceryl-3oleate - Reversed micelles.

\section{INTRODUCTION}

Lipid oxidation is an important cause of decrease of flavour and nutritive value of fats, oil, and oil-containing food (Gunstone and Noris, 1983). To overcome this problem synthetic antioxidants such as butylated hydroxyanisol (BHA) and butylated hydroxytoluene (BHT) are often used. However there is a decline in their usage (Dziezack, 1986) probably due to consumers' preference of natural antioxidants as well as health legislation. For this reason natural antioxidants are used in an increased demand. Some of the natural antioxidants are insoluble or sparingly soluble in edible oils. To disperse water-soluble antioxidants in oil, reversed micelles (RMs) have been used recently (Han et al., 1990; Ock-Sook et al., 1991). RMs are a class of surfactant which aggregates in organic solvents forming structures in which the hydrophilic groups of the surfactant form the central part of the core whereas the hydrophobic moieties form the outer layer of the aggregate. Due to the formation of microenvironments RMs are able to solubilize water and other polar substances in non-polar phases (Ock-Sook et al., 1991; Omar, 1994; Fletcher, 1986; Maja and Björn, 1994 ).

Previous studies on the use of RMs demonstrated the solubilization of ascorbic acid in oil and compared the activity with other antioxidants in the absence of RMs (Han et al., 1990; Ock-Sook et al., 1991). However, the influence of surfactants or RMs themselves on lipid oxidation has not been studied yet.

The objective of this study was to investigate the effect of RMs with different surfactants on the autooxidation and photooxidation of stripped corn oil, and their influence on the activity of antioxidants.

\section{MATERIALS AND METHODS}

\subsection{Materials}

Corn oil stripped of tocopherol was obtained under nitrogen from Acros Organics (Goldenhausen, Germany). $\alpha$-Tocopherol was purchased from Sigma Chemical Co.,St.Louis,MO. Trolox was from Aldrich Chemical Co.(Steinheim ,Germany). The emulsifiers, polyglyceryl-3-oleate (PGO) and methylglucose dioleate (MGO) were a gift from Th. Goldschmidt AG (Essen, Germany) and lecithin (filtrated soybean lecithin, $62 \%$ phospholipids; Sternprime N-10) was provided by Stern Lecithin \& Soja GmbH \& Co KG (Hamburg, Germany).

\subsection{Sample preparation and experimental methods}

\section{Oil sample preparation}

Stripped corn oil samples $(9.5 \mathrm{~g})$ were mixed with $0.5 \mathrm{~g}$ emulsifier and stirred for a 10 minutes; $100 \mu \mathrm{L}$ 
$\mathrm{H}_{2} \mathrm{O}$ were injected stepwise during stirring until clear solution was obtained. Stripped corn oil without addition was taken as a control and compared with the following oil samples: (1) stripped corn oil with added antioxidant (Trolox or $\alpha$-tocopherol), (2) stripped corn oil containing RMs alone and (3) stripped corn oil containing RMs in combination with Trolox or $\alpha$-tocopherol. $\alpha$-Tocopherol or Trolox was introduced in methanol to the empty glass bottles in a final concentration of $100 \mu \mathrm{mol}$ per $\mathrm{kg}$ oil and then was purged with nitrogen gas to evaporate the solvent.

\section{Methods}

Samples were stored in screw capped amber glass bottles at $60{ }^{\circ} \mathrm{C}$ (autooxidation) and for photooxidation on screw capped clear glass bottles at light intensity of $10.7 \mathrm{~K}$ Lux and constant temperature of $25 \pm 1^{\circ} \mathrm{C}$.

Oxidative stability was followed up by measuring primary oxidation products (peroxides) and secondary oxidation products (hexanal).

\section{Peroxide determination}

Was done according to the method of the International Dairy Federation (IDF) for peroxide determination (Shantha and Decker, 1994), which is a spectrophotometric method based on the ability of peroxides to oxidize ferrous ions to ferric.

To prepare the iron (II) chloride solution, $0.4 \mathrm{~g}$ barium chloride dihydrate was dissolved in $50 \mathrm{~mL}$ water. This solution was added slowly and with constant stirring to an iron (II) sulfate solution $(0.5 \mathrm{~g}$ $\mathrm{FeSO}_{4} .7 \mathrm{H}_{2} \mathrm{O}$ dissolved in $50 \mathrm{~mL}$ water). Two milliliters of $10 \mathrm{~N}$ hydrochloric acid was added to the resulting solution. The barium sulphate precipitate was filtered off to give a clear iron (II) solution, which was stored in a brown bottle and kept in dark. To prepare the ammonium thiocyanate solution, $30 \mathrm{~g}$ ammonium thiocyanate was dissolved in $100 \mathrm{~mL}$ water.

To determine the peroxide value, the sample $(0.025 \mathrm{~g})$ was mixed in a glass tube with $9.8 \mathrm{~mL}$ chloroform-methanol (7:3 vol/vol) on a vortex for $10 \mathrm{~s}$. Ammonium thiocyanate soln $(50 \mu \mathrm{L})$ was added, and the sample was mixed on a vortex mixer for $10 \mathrm{~s}$. Then, $50 \mu \mathrm{L}$ iron (II) solution was added, and the sample was mixed on a vortex mixer for $10 \mathrm{~s}$. After 5 min incubation at room temperature, the absorbance was spectrophotometrically read at $500 \mathrm{~nm}$ against a blank that contained all the reagent except the sample. The entire procedure was conducted in subdued light and completed within $10 \mathrm{~min}$.

To construct a standard curve of $\mathrm{Fe}^{+++}$ concentration vs absorbance, a serial of dilutions of standard solution of iron (III) chloride was prepared and treated exactly as before except that the standard is put instead of the sample, then plotting concentration vs absorbance.

The peroxide value, expressed as milliequivalents of $\mathrm{O}_{2} / \mathrm{kg}$ sample, was calculated using the following formula:

$$
\text { Peroxide value }=\frac{\left(A_{s}-A_{b}\right) \times m}{55.84 \times W}
$$

where $A_{s}=$ absorbance of the sample $; A_{b}=$ absorbance of the blank; $m=$ slope, obtained from the calibration curve; $\mathrm{W}=$ sample weight in grams; $55.84=$ atomic weight of iron.

\section{Measurement of hexanal}

Hexanal analysis was carried out according to Frankel et al. (1994) except heating step. Oil (0.5g) samples were measured into special 6-ml headspace vials, sealed with silicon rubber Teflon caps with a crimper. Oils were warmed up at $60^{\circ} \mathrm{C}$ for $15 \mathrm{~min}$. Hexanal was determined within 6 minutes, using a gas chromatograph FTV series 4000, oven 4130, FID 40 (Carlo Erba Instruments) equipped with an HS 250 (Carlo Erba Instruments) and a capillary column DB-1701 FSOT $(30 \mathrm{~mm} x$ $0.32 \mathrm{~mm} I \mathrm{ID}, 1 \mu \mathrm{m}$ film thickness; stationary phase, Cyanopropylphenyl silicon).

The GC conditions were as follows: oven temperature, $65^{\circ} \mathrm{C}$ (isothermal); nitrogen flow rate, $7.5 \mathrm{ml} / \mathrm{min}$; oxygen pressure, $100 \mathrm{kPa}$; hydrogen pressure, $50 \mathrm{kPa}$; splitless Injector temperature, 180 ${ }^{\circ} \mathrm{C}$; detector temperature, $210^{\circ} \mathrm{C}$; injection volume, 1 $\mathrm{ml}$ and water-bath temperature, $60^{\circ} \mathrm{C}$.

After heating, the bottles were pressurized 5 times for 30 seconds before injection.

The method depends on the equilibrium established between the amounts of hexanal present in the gas and liquid phases. Content of hexanal was determined by analyzing quantitatively samples of the gas phase. Peak areas for hexanal were integrated electronically and standardized with known amounts of authentic hexanal. Results were calculated as hexanal in micromoles per kilogram $(\mu \mathrm{mol} / \mathrm{kg})$ of oil.

\section{RESULTS}

The effect of RMs on the oxidation of stripped corn oil was investigated using three different surfactants: lecithin, PGO and MGO. The experiments were carried out under autooxidation and photooxidation with and without addition of the antioxidants $\alpha$-tocopherol and the water soluble analogue Trolox. 


\subsection{Hydroperoxide formation during autooxidation}

After five days the hydroperoxide concentration amounted to $294 \mathrm{mmol} / \mathrm{kg}$ in the control oil (without RMs). In oils containing RMs the hydroperoxide formation was depressed and decreased in the order: $\mathrm{MGO} \cong \mathrm{PGO}>$ lecithin (Fig. 1). Addition of antioxidants caused a considerable decrease in hydroperoxides of all samples either without (control) or in combination with RMs (Fig. 2). Oils containing Trolox showed lower hydroperoxide formation than the corresponding systems with $\alpha$-tocopherol.

\subsection{Inhibition of hydroperoxide formation}

In order to demonstrate the effect of the RMs on the activity of the added antioxidants, the inhibition of hydroperoxide formation was calculated relative to each system control (the hydroperoxide concentration of bulk oil without antioxidant, Lecithin-RMs without antioxidant, PGO-RMs without antioxidant or MGO-RMs without antioxidant). The results indicated that the activity of both antioxidants was reduced in the presence of RMs in comparison with bulk oil without RMs and the same increasing

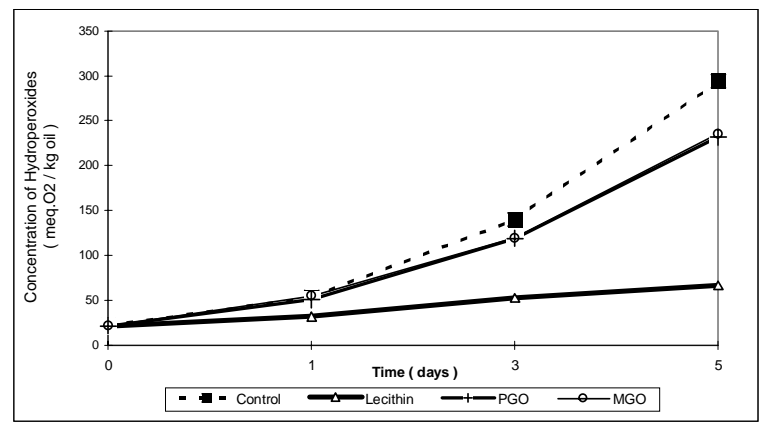

Figure 1

Hydroperoxide formation of autooxidized stripped corn oil, without antioxidants, PGO =Polyglycerol-3-oleate; $\mathrm{MGO}=$ Methylglucose dioleate .

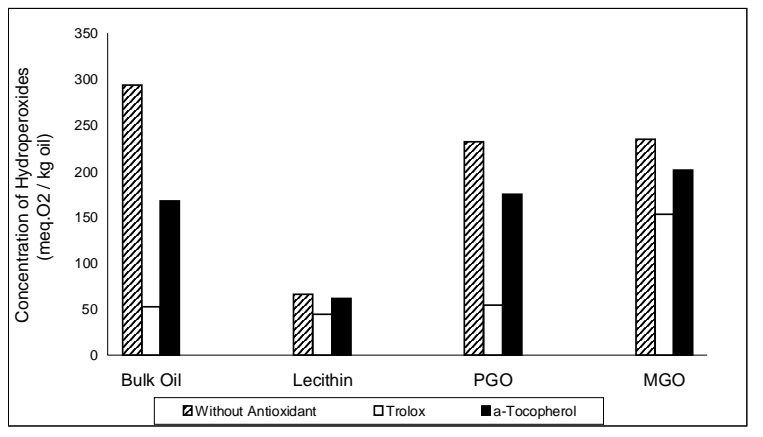

Figure 2

Effect of reversed micelles on hydroperoxide formation of autooxidized stripped corn oil in the presence of antioxidants, PGO =Polyglycerol-3-oleate; $M G O=$ Methylglucose dioleate .

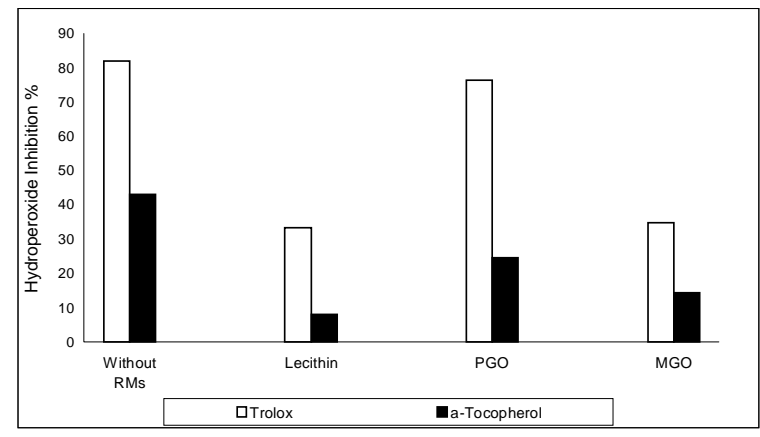

Figure 3

Effect of hydroperoxide inhibition of autooxidized stripped corn oil in the presence of antioxidants. PGO =Polyglycerol-3-oleate; $\mathrm{MGO}=$ Methylglucose dioleate .

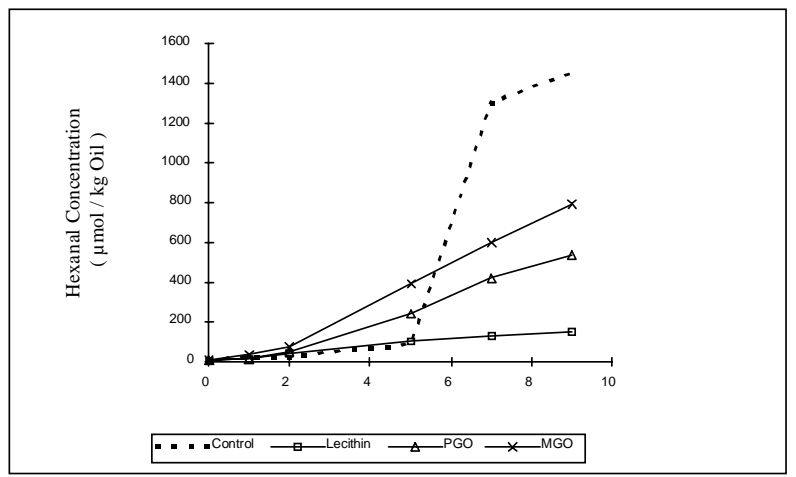

Figure 4

Hexanal Formation of autooxidized stripped corn oil without antioxidants, $\mathrm{PGO}=$ polyglycerol-3-oleate; $\mathrm{MGO}=$ methyl glucose dioleate

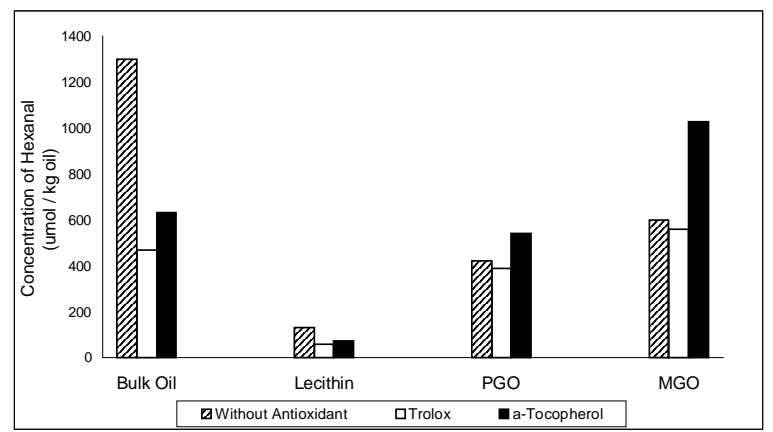

Figure 5

Effect of reversed micelles on hexanal formation of autooxidized stripped corn oil in the presence of antioxidants, $\mathrm{PGO}=$ Polyglycerol-3-oleate; $\mathrm{MGO}=$ Methylglucose dioleate .

order of hydroperoxide inhibition for samples containing $\alpha$-tocopherol or Trolox was followed (lecithin-RMs < MGO-RMs < PGO-RMs bulk oils without RMs; Fig. 3). 


\subsection{Hexanal formation during autooxidation}

Hexanal formation was measured after 7 days as the secondary oxidation products (e.g. hexanal) is usually developed later than the primary one (i.e. hydroperoxides). After 7 days the order of hexanal formation followed the same trend as was observed for hydroperoxide formation: control (bulk oil without RMs) > MGO > PGO lecithin (Fig. 4). The addition of $\alpha$-tocopherol or Trolox in the presence of RMs resulted in a lower hexanal formation in all samples compared to the control after 7 days $(1300 \mu \mathrm{mol} / \mathrm{kg}$; Fig.5).

\subsection{Inhibition of hexanal during autooxidation}

In order to demonstrate the effect of the RMs on the activity of antioxidants, the inhibition of hexanal formation due to the addition of Trolox and $\alpha$-tocopherol was expressed relative to corresponding system control (with or without RMs Fig. 6) exactly as in case of calculating the hydroperoxide inhibition. In all samples the inhibition due to the antioxidant was reduced when compared with the control (bulk oil without RMs). In the case of $\alpha$-tocopherol a prooxidant effect was found in systems containing PGO and MGO RMs.

\subsection{Hydroperoxide formation during photooxidation}

During photooxidation lecithin and PGO RMs caused a decrease of hydroperoxide formation in the oil, whereas MGO RMs showed higher hydroperoxide formation than bulk oil without RMs (Fig. 7). The combination of $\alpha$-tocopherol or Trolox and RMs caused in most oils a significant reduction of the hydroperoxide formation (Fig. 8) compared to the control bulk oil $(108 \mathrm{mmol} / \mathrm{kg})$. Only in the case of $\alpha$-tocopherol in oil containing

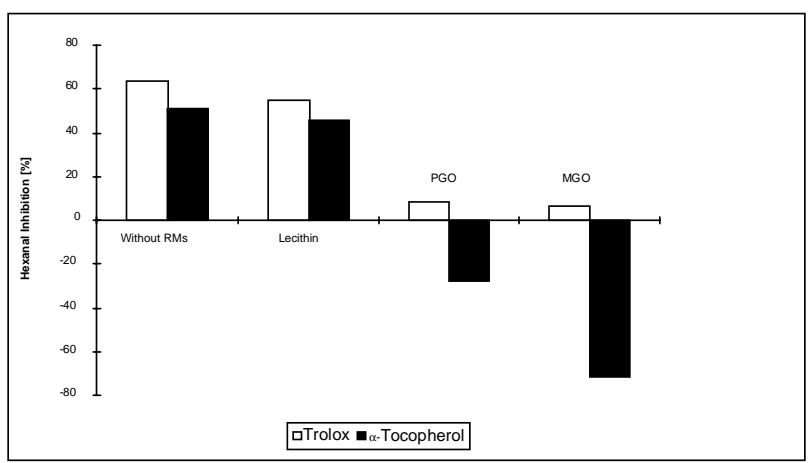

Figure 6

Effect of reversed micelles on hexanal inhibition of autooxidized stripped corn oil in the presence of antioxidants,

$\mathrm{PGO}=$ polyglycerol-3-oleate; $\mathrm{MGO}=$ methyl glucose dioleate .

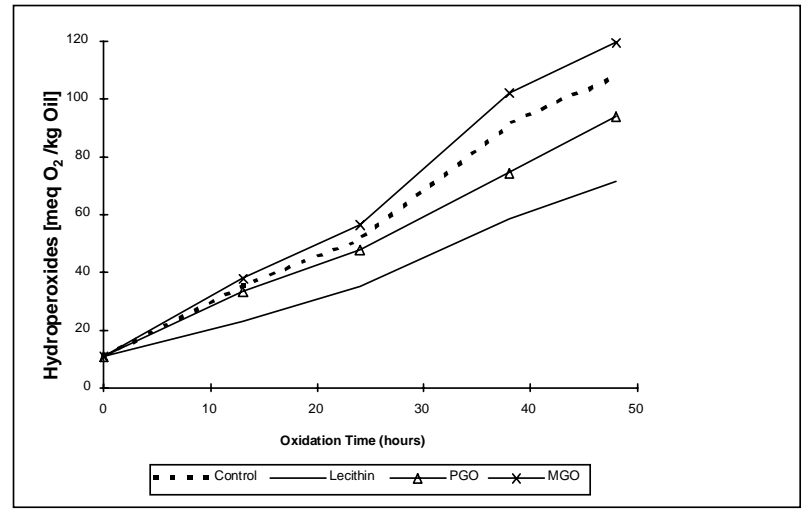

Figure 7

Hydroperoxide formation of photooxidized stripped corn oil without antioxidants, $\mathrm{PGO}=$ polyglycerol-3-oleate; $\mathrm{MGO}=$ methyl glucose dioleate.

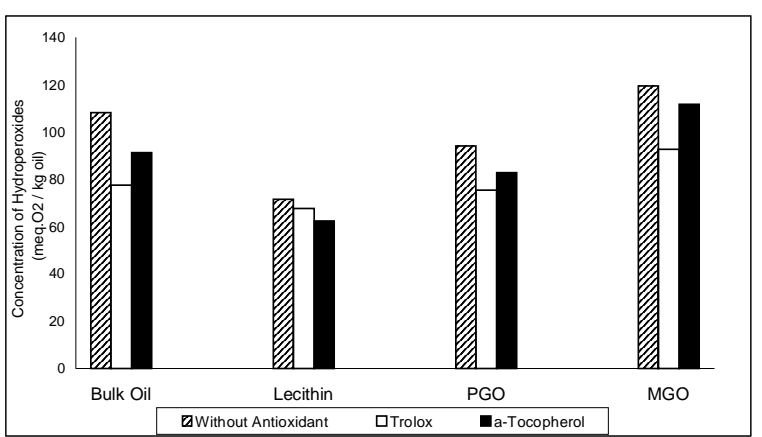

Figure 8

Effect of reversed micelles on hydroperoxide formation of photooxidized stripped corn oil in the presence of antioxidants, PGO =Polyglycerol-3-oleate; MGO = Methylglucose dioleate.

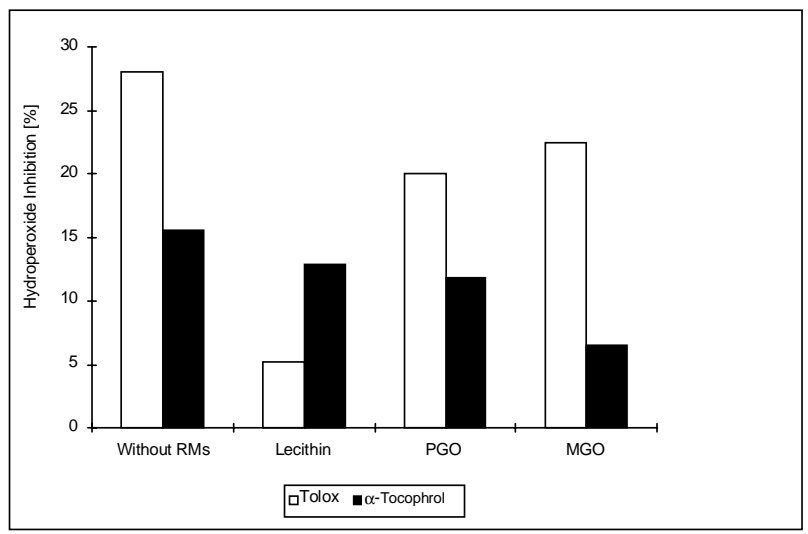

Figure 9

Effect of reversed micelles on hydroperoxide inhibition of photooxidized stripped corn oil in the presence of antioxidants, $\mathrm{PGO}=$ polyglycerol-3-oleate; $\mathrm{MGO}=$ methyl glucose dioleate.

MGO RMs the hydroperoxide formation was slightly enhanced. 


\subsection{Inhibition of hydroperxide formation during photooxidation}

To demonstrate the effect of the RMs on the antioxidant activity during photoxidation, the inhibition of hydroperoxide formation due to the antioxidant was expressed relative to corresponding system (with or without RMs; Fig. 9). The order of hydroperoxide inhibition was MGO RMs > PGO RMs > lecithin RMs when Trolox was added. In the case of $\alpha$-tocopherol the order was reversed. In contrast to the autoxidation experiments $\alpha$-tocopoherol showed higher inhibition of hydroperoxide formation in combination with lecithin than Trolox.

\subsection{Hexanal formation during photooxidation}

After $191 \mathrm{~h}$ the hexanal formation amounted to $561 \mu \mathrm{mol} / \mathrm{kg}$ in the control oil (without RMs). In the case of PGO RMs and lecithin RMs the hexanal formation was reduced, but in the case of MGO an increase in hexanal formation was found (Fig. 10). As shown in Fig. $11 \alpha$-tocopherol caused significless $(p<0.05)$ hexanal formation than Trolox when added to bulk oil or lecithin samples, but in the case of PGO RMs after $192 \mathrm{~h}$ the difference was not significant. Oils containing MGO RMs in combination with Trolox caused a clear decrease in hexanal formation compared to bulk oil while in combination with $\alpha$-tocopherol a large increase in hexanal formation was found.

\subsection{Inhibition of hexanal formation during photooxidation}

In order to demonstrate the effect of the RMs on the antioxidant activity, the inhibition of hexanal formation due to the antioxidant addition was expressed relative to the corresponding system (with or without RMs; Fig. 12). The activity of Trolox

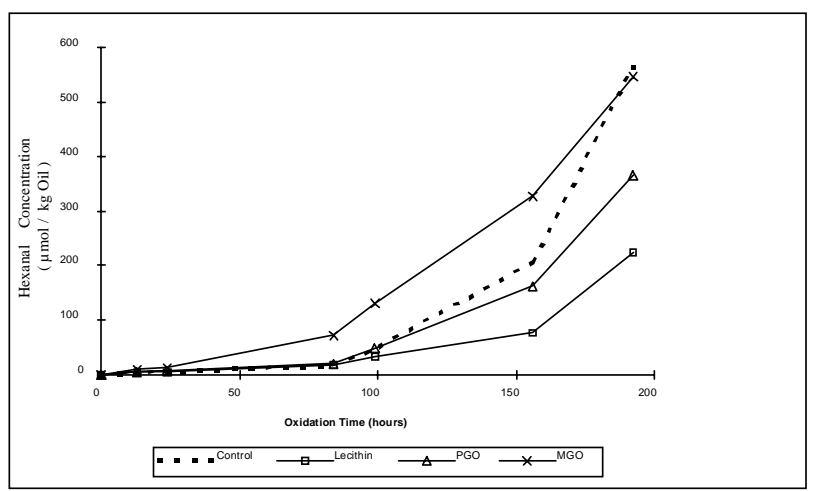

Figure 10

Effect of reversed micelles on hexanal formation of photooxidized stripped corn oil without antioxidants, $\mathrm{PGO}=$ polyglycerol-3oleate; MGO=methyl glucose dioleate.

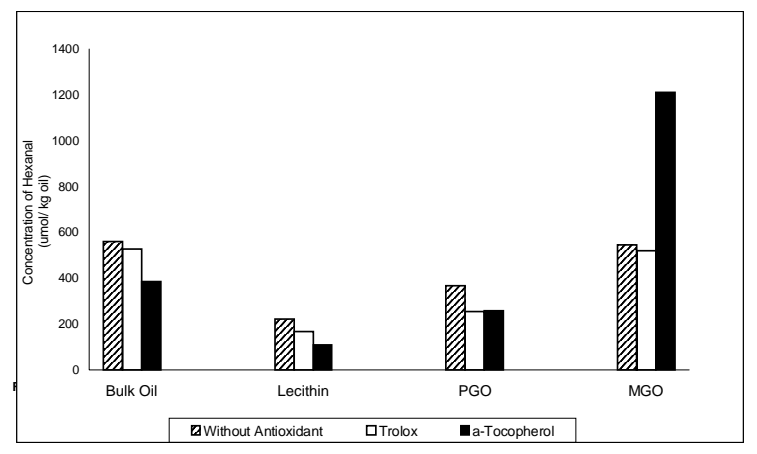

Figure 11

Effect of reversed micelles on hexanal formation of photooxidized stripped corn oil in the presence of antioxidants, PGO =Polyglycerol-3-oleate; $\mathrm{MGO}$ = Methylglucose dioleate.

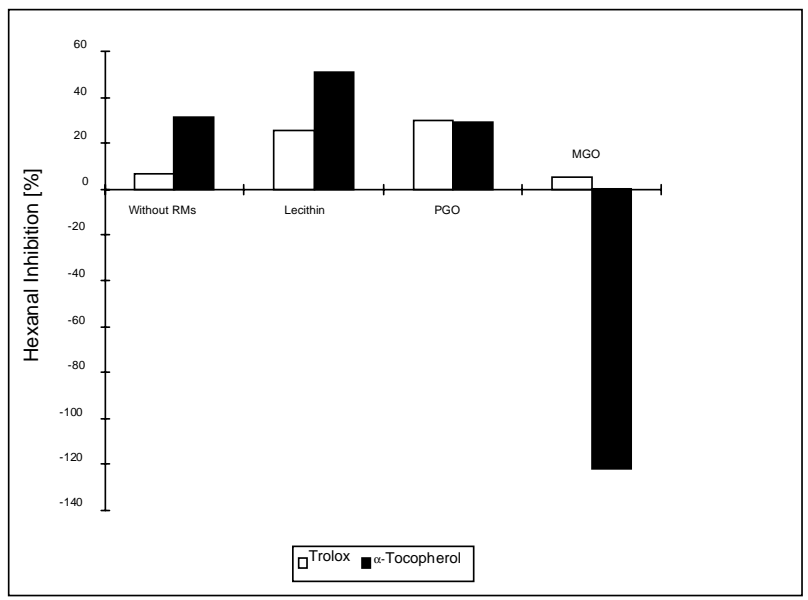

Figure 12

Effect of reversed Micelles on hexanal Inhibition of photooxidized stripped corn oil in the presence of antioxidants, $\mathrm{PGO}=$ polyglycerol-3-oleate; $\mathrm{MGO}=$ methyl glucose dioleate .

decreased in the order PGO RMs > lecithin RMs >> MGO RMs whereas the inhibiting activity of $\alpha$-tocopherol decreased in the order lecithin RMs > Isolan GO33 RMs and in the presence of Isolan DO RMs a high prooxidative effect was found.

\section{DISCUSSION}

It was shown that RMs formed by different surfactants caused a lower degree of lipid oxidation compared to the control bulk oil except in the case of MGO RMs in photooxidation. The stabilizing effect of RMs toward oxidation may be explained, firstly by the presence of traces of water in the RMs that may cause hydration of metal ions in the core of RMs and thereby decreasing their effectiveness towards hydrogen bonds of hydroperoxide (Omar, 1994) and 
secondly, at the same time RMs form separate microenvironments for metal ions which may reduce their mobility. By contrast, at higher water activity and moisture content increased metal mobility and decreasing oil viscosity may cause accelerated oxidation Labuza and Dugan, 1971).

The stronger activity of lecithin RMs compared to the other emulsifiers in depressing autooxidation can be attributed to the phosphatidyl ethanolamine and phosphoric acid moieties of lecithin. The nitrogen base of the phosphatidyl ethanolamine group is so weak that the hydroxyl group of the phosphoric acid remains mostly free, hence hydrogen donation is greatly facilitated. At the same time phosphoric acid is able to chelate metals (Evans et al., 1954; Swift et al., 1974; Evans et al., 1951; Cowan et al., 1962).

In bulk oil without RMs micelles Trolox showed higher antioxidant activity than $\alpha$-tocopherol under autooxidation. This effect was attributed to the low oil solubility of Trolox which might result in an accumulation at the air-oil interface (Frankel et al., 1994; Uri, 1961; Porter et al., 1989).

In photooxidation the order of antioxidant activity between $\alpha$-tocopherol and Trolox was partly reversed, i.e., $\alpha$-tocopherol was more active in inhibiting hexanal formation. This observation is in agreement with the study of Iliou et al., 1992 who reported that $\alpha$-tocopherol has been found to inhibit the latter steps of photooxidation involving radical mechanism.

Whether the reduced activity of Trolox in oils containing RMs be attributed to the ability of the RMs to host polar molecules is not clear, as we observed the same decreasing effect for $\alpha$-tocopherol in the presence of RMs.

The inhibiting effect of Trolox or $\alpha$-tocopherol was found to be stronger in the presence of PGO RMs and MGO RMs than in the presence of lecithin RMs. This difference may be due to the higher oxidation stability of systems containing lecithin RMs, thus, the additional benefit due to the antioxidant additive is rather small. By contrast, PGO RMs and PGM RMs caused lower oxidation stability.

This study demonstrated that RMs are not inert carriers for polar compounds in non polar solvents, but may affect the stability of oils themselves. Further, the activity of added compounds (i.e. antioxidant) can be influenced via different mechanisms by RMs.

\section{REFERENCES}

Cowan, J.C.; Cooney, P. M. and C.D. Evans (1962) . Citric acid: Inactivating agent for metals or acidic synergist in edible fats?. J. Am. Oil Chemists'Soc. 39, 6 - 9.

Dziezak, J.D. (1986). Preservatives :Antioxidants. Food Technol. 40, 94 - 102.

Evans C.D.; Schwab, A.W.; Moser, H.A.; Hawley J.E. and Melvin E.H. (1951). The flavor problem of soybean oil. VII. Effect of trace metals. J. Am. Oil Chemists'Soc. 28, $68-73$.

Evans, C.D.; Cooney, P.M.; Scholfield C.R.and Hutton H.J. (1954). Soybean "lecithin" and its fractions as metal-inactivating agents. J. Am. Oil Chemists'Soc. 31, $295-297$.

Fletcher, P. D. I. (1986). The partitioning of solutes between water-in-oil microemulsions and conjugate aqueous phases. J.Chem. Soc. Faraday Trans. 1, 82, 2651-2664.

Frankel, E. N.; Shu-Wen Huang; Joseph Kanner,and Bruce German (1994). Interfacial phenomena in the evaluation of antioxidants: bulk oils vs emulsions. J.Agric. Food Chem. 42, 1054-1059.

Gunstone,F.D. and Noris , F.A. (1983) "Lipids in Foods" Pergamon Press, New York.

Han, D.; YI, O.S. and Shin, H.K. (1990). Antioxidative effect of ascorbic acid solubilized in oils via reversed micelles. J. Food Science 55, 247-248.

Iliou, J.-P.; Jourd'Heuil, D.; Robin, R.; Serkiz,B. ; Guivarch, P.; Volland J.-P. and Vilaine,J.-P. (1992). Kinetics of photoperoxidation of arachidonic acid: molecular mechanisms and effects of antioxidants. Lipids 27, 959-967.

Labuza , T.P. and Dugan, L. R. (1971). Kinetics of Lipid Oxidation in Food. CRC Crit.Rev.Food Technol. 2, 355.

Maja Jakobsson and Björn Sivik (1994). Oxidative stability of fish oil included in a microemulsion. J.Despersion Science and Technol. 15, 611-619.

Ock-Sook ,Daeseok Han and Hyun-Kyung Shin (1991). Synergistic antioxidative effects of tocopherol and ascorbic acid in fish oil/lecithin/water system. J. Am. Oil Chemists'Soc. 68, 881-883.

Omar A. Elseoud (1994). Formation and Some Relevant Properties, In: Reversed Micelles and Water -in - Oil Microemulsion (Ed JAI Press Inc.), P. 2-30.

Porter ,W.L.; Black ,E.D.; Drolet, A.M. (1989). Use of polyamide oxidative flourescencetest on lipid emulsion:contrast in relative effectiveness of antioxidants in bulk versus dispersed systems. J. Agric. Food Chem. 37, 615-623.

Shantha, N. C. and Decker, E.A. (1994). Rapid, Sensitive, Iron-Based spectrophotometric methods for determination of peroxide values of food lipids. J. of AOAC International 77, 421-424.

Swift,C.E.; Rose, W.G. and Jamies G.S. (1974). In Oil and Soap 19, 176.

Uri, N. (1961). Mechanism of antioxidation. In Autoxidation and Antioxidants. Lundberg, W.O. , Ed. Interscience: New York; Vol.1, Chapter 4. 